OPEN ACCESS

Edited by:

Fabienne Brilot

The University of Sydney, Australia

Reviewed by:

Amy Kunchok

Mayo Clinic, United States

Marco A. Lana-Peixoto,

Federal University of Minas

Gerais, Brazi

${ }^{*}$ Correspondence:

Friedemann Paul

friedemann.paul@charite.de

tThese authors have contributed equally to this work and share senior

authorship

Specialty section: This article was submitted to Applied Neuroimaging, a section of the journa Frontiers in Neurology

Received: 23 September 2019

Accepted: 24 August 2020

Published: 30 September 2020

Citation:

Schmidt FA, Chien C, Kuchling J, Bellmann-Strobl J, Ruprecht K,

Siebert N, Asseyer S, Jarius S,

Brandt $A U$, Scheel $M$ and Paul $F$ (2020) Differences in Advanced Magnetic Resonance Imaging in MOG-IgG and AQP4-IgG Seropositive Neuromyelitis Optica Spectrum Disorders: A Comparative Study.

Front. Neurol. 11:499910. doi: 10.3389/fneur.2020.499910

\section{Differences in Advanced Magnetic Resonance Imaging in MOG-IgG and AQP4-IgG Seropositive Neuromyelitis Optica Spectrum Disorders: A Comparative Study}

Felix A. Schmidt ${ }^{1,2,3,4}$, Claudia Chien ${ }^{1}$, Joseph Kuchling ${ }^{1,2}$, Judith Bellmann-Strobl ${ }^{1,5}$, Klemens Ruprecht ${ }^{2,3}$, Nadja Siebert ${ }^{1,5}$, Susanna Asseyer ${ }^{1,5}$, Sven Jarius ${ }^{6}$, Alexander U. Brandt ${ }^{1,5,7}$, Michael Scheel ${ }^{1,4,8 t}$ and Friedemann Paul ${ }^{1,2,5 * t}$

\footnotetext{
${ }^{1}$ NeuroCure Clinical Research Center, Charité - Universitätsmedizin Berlin, Corporate Member of Freie Universität Berlin, Humboldt-Universität zu Berlin, and Berlin Institute of Health, Berlin, Germany, ${ }^{2}$ Department of Neurology, Charité Universitätsmedizin Berlin, Corporate Member of Freie Universität Berlin, Humboldt-Universität zu Berlin, and Berlin Institute of Health, Berlin, Germany, ${ }^{3}$ Clinical and Experimental Multiple Sclerosis Research Center, Department of Neurology, Charité - Universitätsmedizin Berlin, Berlin, Germany, ${ }^{4}$ Berlin Institute of Health, Berlin, Germany, ${ }^{5}$ Experimental and Clinical Research Center, Max Delbrueck Center for Molecular Medicine and Charité - Universitätsmedizin Berlin, Corporate Member of Freie Universität Berlin, Humboldt-Universität zu Berlin, and Berlin Institute of Health, Berlin, Germany, ${ }^{6}$ Division of Molecular Neuroimmunology, Department of Neurology, University Hospital Heidelberg, Heidelberg, Germany, ${ }^{7}$ Department of Neurology, University of California, Irvine, Irvine, CA, United States, ${ }^{8}$ Department of Neuroradiology, Charité -

Universitätsmedizin Berlin, Berlin, Germany
}

Aims: To explore differences in advanced brain magnetic resonance imaging (MRI) characteristics between myelin oligodendrocyte (MOG) immunoglobulin (lgG) and aquaporin-4 (AQP4) lgG seropositive (+) neuromyelitis optica spectrum disorders (NMOSD).

Methods: 33 AQP4-IgG and 18 MOG-IgG seropositive NMOSD patients and 61 healthy control $(\mathrm{HC})$ subjects were included. All 112 participants were scanned with the same standardized MRI-protocol on a 3-Tesla MRI-scanner. Brain volume and diffusion tensor imaging (DTI) parameters were assessed.

Results: MOG-IgG+ patients showed reduced parallel diffusivity within white matter tracts compared to HC whereas AQP4-IgG+ showed no significant brain parenchymal damage in DTI analysis. AQP4-IgG+ patients showed reduced whole brain volumes and reduced volumes of several deep gray matter structures compared to HC whereas MOG-IgG + patients did not show reduced brain or deep gray matter volumes compared to $\mathrm{HC}$.

Conclusions: Microstructural brain parenchymal damage in MOG-lgG+ patients was more pronounced than in AQP4-IgG+ patients, compared with $\mathrm{HC}$, whereas normalized brain volume reduction was more severe in AQP4-lgG+ patients. Longitudinal imaging studies are warranted to further investigate this trend in NMOSD. Our results suggest 
that MOG-lgG+ and AQP4-lgG+ NMOSD patients differ in cerebral MRI characteristics. Advanced MRI analysis did not help to differentiate between MOG-lgG+ and AQP4-lgG+ patients in our study.

Keywords: NMOSD, AQP4, MOG, MRI, DTI, advanced imaging

\section{INTRODUCTION}

Neuromyelitis optica spectrum disorders (NMOSD) are autoimmune diseases that present with longitudinally extensive transverse myelitis (LETM) and or optic neuritis (ON). They can also present with area postrema, diencephalic, cerebral or acute brainstem syndromes (1-6). The proof of serum autoantibodies directed against aquaporin-4 (AQP4-IgG) in around $80 \%$ of cases established NMOSD as a distinct disease from multiple sclerosis (MS) (7-10). In a subgroup of AQP4-IgG negative NMOSD patients serum antibodies targeting the myelin oligodendrocyte glycoprotein (MOG-IgG) were detected (11-16). MOG-IgG+ patients with clinical and neuroimaging characteristics of NMOSD are currently discussed as a different disease entity (17-23). Cortical encephalitis and seizures or cranial nerve involvement have also been reported in MOG-IgG+ patients (24-27).

While brain atrophy and microstructural tissue damage occur in MS from earliest disease stages (28-30), non-conventional MRI studies have shown conflicting results in NMOSD (31-35), and advanced imaging, such as diffusion tensor imaging (DTI) analysis, have not been reported from MOG-IgG+ patients.

Thus, the goal of our study was to further investigate possible MRI differences between MOG-IgG+ and AQP4-IgG+ mediated pathology in NMOSD patients and to compare these effects to a group of HCs.

\section{MATERIALS AND METHODS}

\section{Study Participants and Controls}

Data for this cross-sectional study were extracted from an ongoing longitudinal observational study following patients with NMOSD and HCs performed at the NeuroCure Clinical Research Center, Charité Universitaetsmedizin Berlin.

Inclusion criteria were diagnosis of NMOSD according to the international consensus diagnostic criteria for NMOSD 2015 (36) or positive proof of MOG-IgG serum antibodies in a live cell-based assay and an associated demyelinating CNS disease with a clinical phenotype equivalent to NMOSD diagnosis criteria in patients over 18 years (17). In this regard, we treated MOG-IgG positivity as equivalent to positive AQP4-IgG for fulfilling the diagnostic criteria. All MOGIgG + patients met the currently proposed criteria for MOG encephalomyelitis (26). Exclusion criteria were a relapse within 3 months prior to MRI examination. Patients from the outpatient clinics of the Experimental and Clinical Research Center, NeuroCure Clinical Research Center and from the Department of Neurology, Charité - Universitätsmedizin Berlin were screened for eligibility.

\section{Ethics Statement}

The study was approved by the Charité-Universitätsmedizin Berlin ethics committee (EA1/041/14) and was conducted in accordance to the Declaration of Helsinki in its currently applicable version and applicable German laws. All participants gave written informed consent to participate in the study.

\section{CLINICAL DATA}

\section{Clinical Assessment}

The expanded disability status scale (EDSS) and further clinical characteristics were assessed on the day the MRI scan was performed as part of a study protocol. All patients included were enrolled into a prospective observational cohort study of NMOSD at NeuroCure Clinical Research Center and received a MRI scan annually as part of this cohort study. All patients were examined with the same standardized MRI protocol at the same MRI scanner for study purposes. From the majority of patients no MRI at disease onset was available because most of the included patients were referred from other hospitals and the department of neurology - Charité - Universitätsmedizin Berlin.

\section{MRI Acquisition Protocol}

The multimodal brain MRIs all were acquired from a $3 \mathrm{~T}$ MRI (MAGNETOM Trio Tim, Siemens, Erlangen, Germany) and included a 3-dimensional T1-weighted magnetization prepared rapid acquisition gradient echo (MPRAGE) sequence $(1 \times 1 \times$ $1 \mathrm{~mm}$ resolution, $\mathrm{TR}=1,900 \mathrm{~ms}, \mathrm{TE}=3.03 \mathrm{~ms}$ ), a 3-dimensional fluid attenuated inversion recovery (3D FLAIR) sequence (1 $\times 1 \times 1 \mathrm{~mm}$ resolution, $\mathrm{TR}=6,000 \mathrm{~ms}, \mathrm{TE}=388 \mathrm{~ms})$ and a single-shot echo planar imaging DTI sequence $(\mathrm{TR} / \mathrm{TE}=$ $7,500 / 86 \mathrm{~ms} ;$ FOV $=240 \times 240 \mathrm{~mm}$; matrix $96 \times 96,61$ slices no gap, slice thickness $2.3 \mathrm{~mm}, 64$ non-collinear directions, b-value $=1,000 \mathrm{~s} / \mathrm{mm}^{2}$ ).

\section{MRI-Data Post-processing Brain and Deep Gray Matter Volumes}

Gray and white matter volumes were calculated from MPRAGE scans, after lesion filling, using FSL SIENAX for normalized whole brain gray matter and white matter and with FSL FIRST for deep gray matter volumes (37-40).

\section{DTI PARAMETERS}

FSL DTIFIT (41) was used for DTI data processing, which included brain extraction and correction for eddy current distortions. Fractional anisotropy (FA) and parallel diffusivity were calculated by fitting a tensor model to the diffusion data using the tools from FSL. All data were then further processed with the tract based spatial statistics (TBSS) $(42,43)$. 


\section{Statistical Analysis}

Statistical analyses was were performed with $\mathrm{R}$ version 3.3.0 using the packages geepack and ggplot2 (44). Differences in demographics and clinical characteristics between patients and HC were tested using Welch's two-sample $t$-test. Differences in gender, clinical phenotype and immunosuppressive therapy were tested with the Fisher Exact Test. Group differences (brain volume and DTI measures) were analyzed using a linear regression model, including age, female sex and disease duration as a covariate. A $p$-value of $<0.05$ was considered significant. Due

TABLE 1 | Demographics and clinical characteristics of AQP4-lgG+ and MOG-IgG+ patients.

\begin{tabular}{lccc}
\hline Parameter & AQP4 $(\boldsymbol{n}=\mathbf{3 3})$ & MOG $(\boldsymbol{n}=\mathbf{1 8})$ & $\boldsymbol{p}$-value \\
\hline Sex (f/m) & $30 / 3$ & $11 / 7$ & $\mathbf{0 . 0 2}$ \\
Age in years & $50.3 \pm 13.6(24-72)$ & $44.3 \pm 12.2(22-59)$ & 0.11 \\
EDSS & $4(0-7)$ & $2(1-6)$ & $\mathbf{0 . 0 1}$ \\
Annualized Relapse Rate & $0.8 \pm 1.2$ & $1.3 \pm 1.2$ & 0.28 \\
Total number of relapses & $3.6 \pm 2.1$ & $3.7 \pm 2.5$ & 0.89 \\
Disease Duration in years & $8 \pm 7$ & $8 \pm 11$ & 1.0 \\
Immunosuppressive Therapy & $28(85 \%)$ & $14(78 \%)$ & 0.70 \\
Clinical Phenotype & $14(42 \%)$ & $5(28 \%)$ & 0.37 \\
LETM + bilateral ON & & & \\
Bilateral ON & $3(9 \%)$ & $4(22 \%)$ & 0.23 \\
LETM only & $12(36 \%)$ & $3(17 \%)$ & 0.20
\end{tabular}

F, female; M, Male; EDSS, Expanded Disability Status Scale; LETM, Iongitudinal extensive transverse myelitis; ON, optic neuritis. EDSS is presented as median (range). Age, annual relapse rate, total number of previous attacks and disease duration are presented as mean \pm standard deviation. Clinical phenotype and immunosuppressive therapy are presented as total numbers and in \%. to the exploratory nature of the study, no correction for multiple comparisons was made.

\section{RESULTS}

From the 51 included patients, 33 had a diagnosis of AQP4IgG seropositive NMOSD according to the 2015 IPND diagnostic criteria (36). From the 18 patients with positive MOG-IgG serology, five patients fulfilled the criteria for AQP4-IgG seronegative NMOSD. Another 13 patients were tested positive for serum MOG-IgG and had recurrent $\mathrm{ON}$ or at least one episode of LETM, but did not formally fulfill the 2015 IPND criteria for NMOSD. Demographics and clinical characteristics of AQP4-IG+ and MOG-IgG+ patients are summarized in Table $\mathbf{1 .}$ AQP4-IgG+ and MOG-IgG+ patients showed differences in sex and EDSS score, with no significant difference in age, disease duration, annualized relapse rate and further clinical characteristics (see Table 1).

\section{Brain Volume Analysis}

AQP4-IgG+ patients showed a trend for reduced normalized whole brain volumes compared to $\mathrm{HC}(p=0.087$, see Figure 1). There was no difference between AQP4-IgG+ patients, MOG-IgG+ patients and HC normalized gray matter volumes and white matter volumes.

\section{Brain Volume Analysis of Deep Gray Matter Structures}

MOG-IgG+ patients did not show any volume reduction in deep gray matter structures compared to HC. A significant volume reduction for $\mathrm{AQP} 4-\mathrm{IgG}+$ patients compared to $\mathrm{HC}$ was found in the putamen $(p=0.035$, see Figure 2$)$, the thalamus ( $p=$ 0.019 , see Figure 3 ) and the pallidum ( $p=0.008$, see Figure 4$)$.

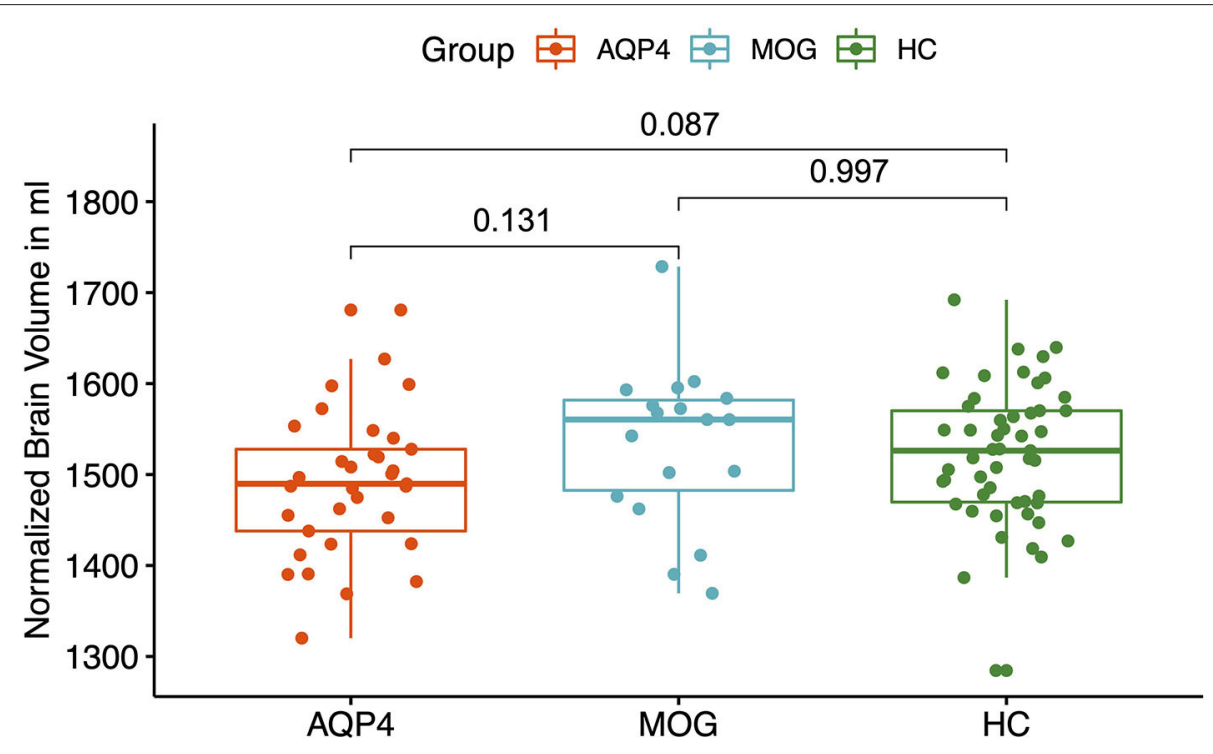

FIGURE 1 | Normalized Brain Volumes. Y-axis: normalized brain volume in ml. X-axis: patient groups and healthy controls. AQP4, Aquaporin-4-IgG+ patients; HC, healthy control; MOG, Myelin oligodendrocyte glycoprotein lgG+ patients. 


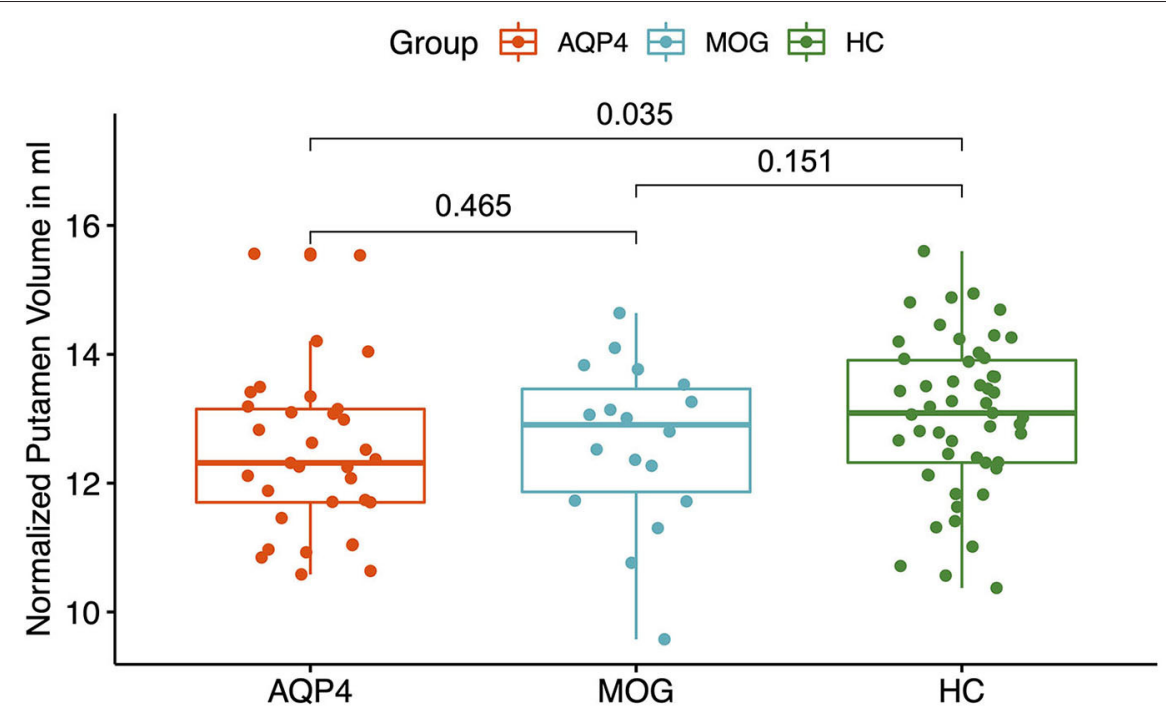

FIGURE 2 | Y-axis: Normalized putamen volume in ml. X-axis: patient groups and healthy controls. AQP4, Aquaporin-4-IgG+ patients; HC, healthy control; MOG, Myelin oligodendrocyte glycoprotein lgG+ patients.

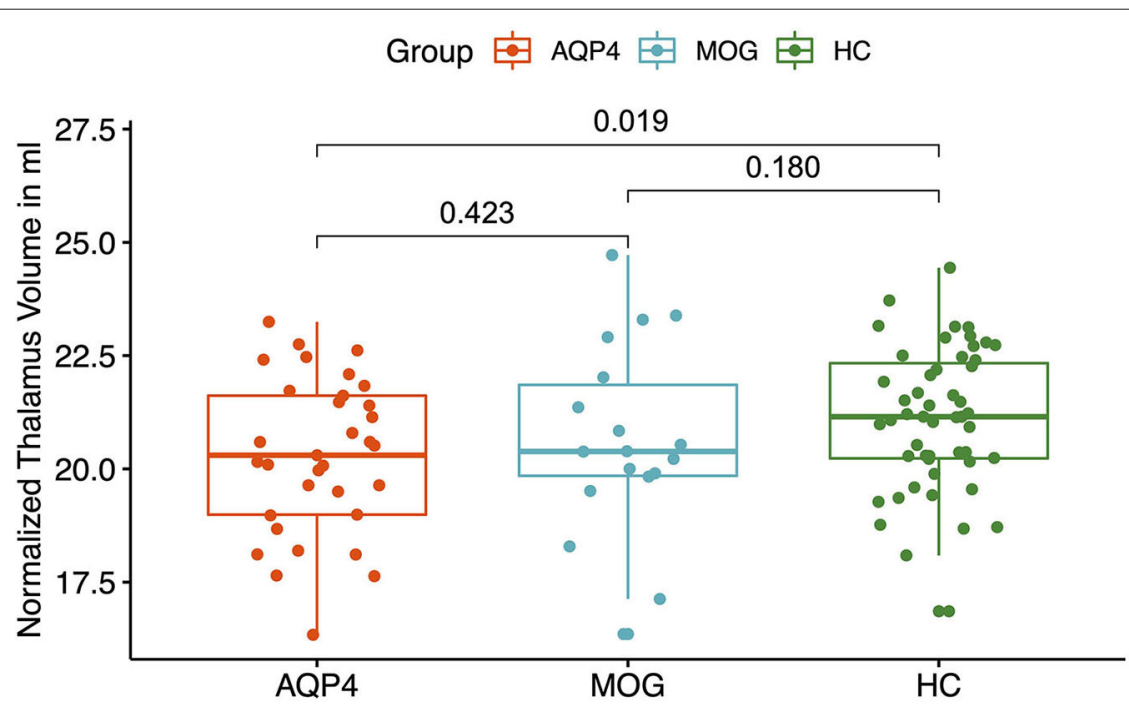

FIGURE 3 | Y-axis: Normalized thalamus volume in ml. X-axis: patient groups and healthy controls. AQP4, Aquaporin-4-lgG+ patients; HC, healthy control; MOG, Myelin oligodendrocyte glycoprotein lgG+ patients.

\section{Diffusion Tensor Imaging}

MOG-IgG+ patients showed reduced parallel diffusivity compared to HC while AQP4-IgG+ patients showed no difference in parallel diffusivity compared to HC (see Figure 5). No difference was found between AQP4-IgG+ and MOG-IgG+ patients or $\mathrm{HC}$ in fractional anisotropy (FA), mean diffusivity, or radial diffusivity measures.

\section{DISCUSSION}

How intracranial volume and brain parenchymal damage differ between MOG-IgG+ and AQP4-IgG+ patients has not been investigated in detail before.
MOG-IgG seropositive patients with clinical and neuroimaging features of NMOSD are currently under discussion as being a distinct disease entity (45-51). There are few studies investigating MRI findings of MOG-IgG+ patients, likely due to its recent discovery and rare prevalence $(18,28,46-48)$.

This is the first study that compares advanced MRI analysis of a larger cohort of MOG-IgG+ patients against AQP4-IgG+ patients along with HC. Other studies demonstrated that MRI brain lesion distribution criteria may help to distinguish MOG$\mathrm{IgG}+$ and $\mathrm{AQP} 4-\mathrm{IgG}+$ patients from multiple sclerosis patients $(28,32,45,47,48,52)$. However, these studies did not analyze differences in brain volume and brain parenchymal damage. 


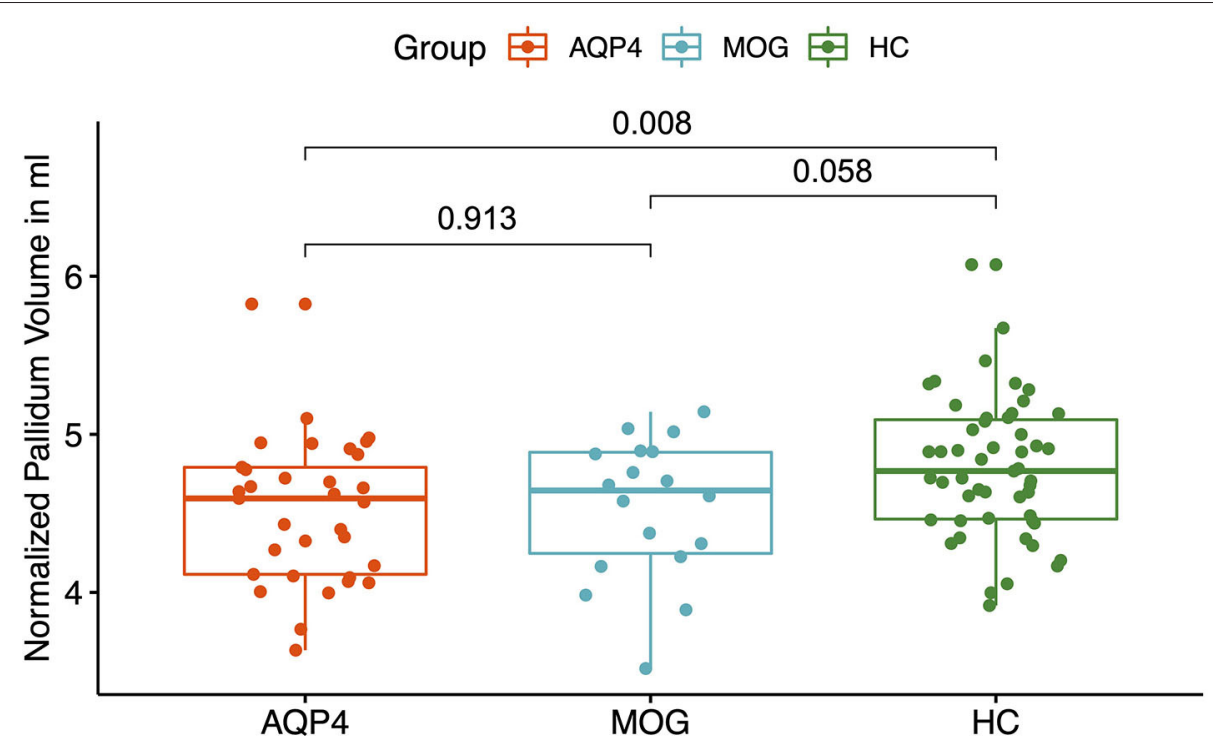

FIGURE 4 | Y-Axis: Normalized pallidum volume in ml. X-axis: patient groups and healthy controls. AQP4, Aquaporin-4-IgG+ patients; HC, healthy control; MOG, Myelin oligodendrocyte glycoprotein $\lg G+$ patients.

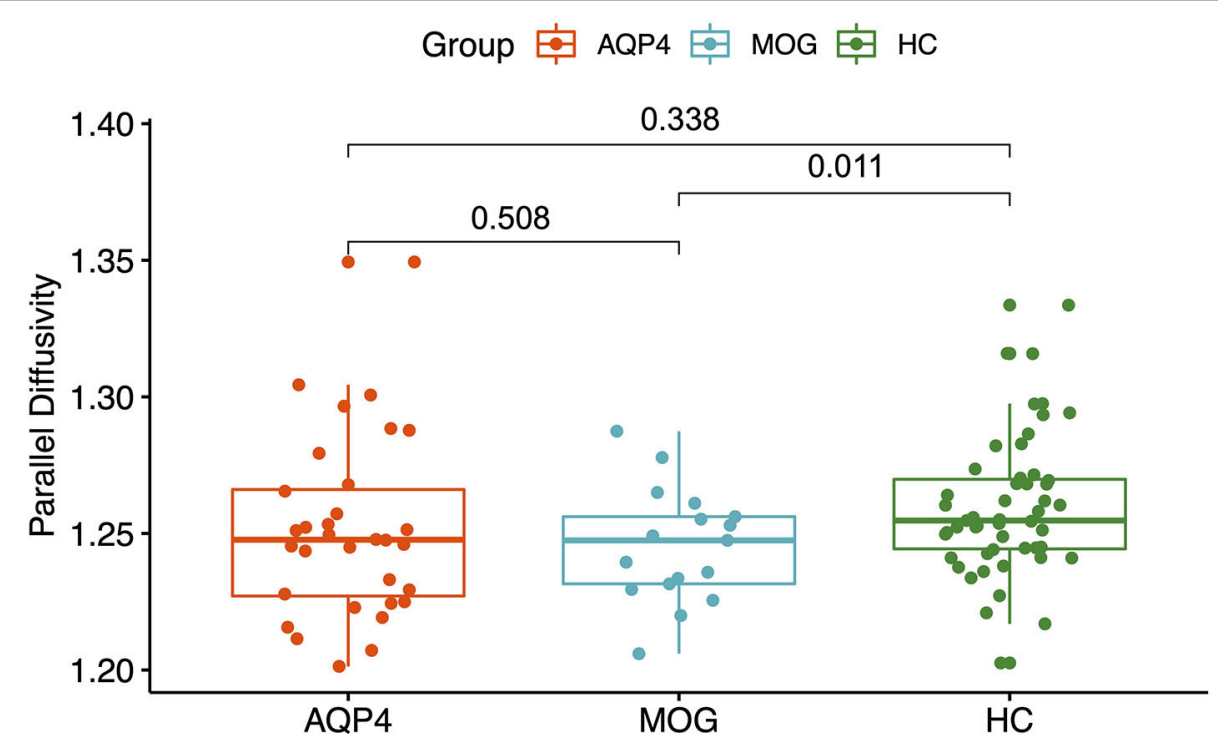

FIGURE 5 | Y-axis: Parallel diffusivity. X-axis: patient groups and healthy controls. AQP4, Aquaporin-4-lgG+ patients; HC, healthy control; MOG, Myelin oligodendrocyte glycoprotein IgG+ patients.

Interestingly, in our brain volume analyses and DTI analysis no significant differences were found between MOG-IgG+ and AQP4-IgG+ patients, although MOG-IgG+ patients showed a reduced parallel diffusivity compared to HC. Our findings of reduced parallel diffusivity in white matter tracts of MOG-IgG+ patients compared to $\mathrm{HC}$ are complemented by previous studies investigating C57BL/6 mice with MOG-induced experimental autoimmune encephalomyelitis (EAE) that showed significant parallel diffusivity reduction within optic nerves (53) and within the spinal chord (54) with significant associations to
EAE clinical scores and greater amounts of axonal damage as confirmed by quantitative staining. Decreased parallel diffusivity within the optic nerves of human patients after acute optic neuritis $(55,56)$ further corroborated the notion that decreased parallel diffusivity may be associated with various mechanisms of axonal damage, such as Wallerian degeneration, and diffuse axonal injury (57) that might have similarly occurred within the white matter of our MOG-IgG+ patients. Axonal loss and atrophy might lead to a bulk reduction of the intraaxonal volume and associated anisotropic diffusion profile 
(58). However, the exact biophysical mechanisms of reduction in parallel diffusivity still remain uncertain and further inand ex-vivo studies are highly warranted to elucidate the exact associations between parallel diffusivity alterations and potentially corresponding axonal damage in patients with MOG-IgG+ encephalomyelitis.

AQP4-IgG+ patients showed a trend for a reduced normalized whole brain volume and a reduced volume of the putamen, pallidum and thalamus compared to $\mathrm{HC}$. In a previous study, a smaller number of AQP4-IgG+ patients from our NMOSD cohort was analyzed and no significant brain volume reduction was found compared to $\mathrm{HC}$ with no brain parenchymal damage $(34,59)$. The differences could be explained by a statistical power problem, since the AQP4-IgG+ patient cohort was smaller.

Several measures were taken to establish a homogeneous study cohort to permit a comparison of these heterogeneous autoimmune disorders. Firstly, only antibody seropositive patients were included. Also, each patient was examined with the same standardized MRI protocol at the same MRI scanner. MRI data was evaluated by the same experienced neuroradiologist blinded to the patients' diagnosis. Further, the current study was restricted to mainly Caucasian patients with NMOSD given that ethnicity likely interacts with NMOSD pathogenicity with regard to possible genetic influence (i.e., Asian opticospinal MS) (60). Finally, AQP4-IgG+ and MOG-IgG+ patients were close in age where there was no significant difference in the means, thus reducing chances of finding differences in brain volume based on age.

A limitation of our study, and most of the previous imaging NMOSD studies, is the limited sample size due to the prevalence of this rare disease, especially in Caucasians (61). One possible solution to this fact, in a rare disease like MOG-IgG+ NMOSD, would be to compile available data in an international study group and pool imaging data in a common database for analysis in multicenter studies. This would allow for the evaluation of brain damage in NMOSD patients with enhanced statistical power. Another limitation are differences in sex and EDSS score between MOG-IgG+ and AQP4-IgG+ patients in our study. Both variables might have an impact on the MRI results.

\section{REFERENCES}

1. Jarius S, Wildemann B, Paul F. Neuromyelitis optica: clinical features, immunopathogenesis and treatment. Clin Exp Immunol. (2014) 176:14964. doi: $10.1111 /$ cei.12271

2. Kleiter I, Gahlen A, Borisow N, Fischer K, Wernecke K, Hellwig K, et al. Apheresis therapies for NMOSD attacks: a retrospective study of 207 therapeuthic interventions. Neurol Neuroimmunol Neuroinflamm. (2018) 5:e504. doi: 10.1212/NXI.0000000000000504

3. Kremer L, Mealy M, Jacob A, Nakashima I, Cabre P, Bigi S, et al. Brainstem manifestations in neuromyelitis optica: a multicenter study of 258 patients. Mult Scler. (2014) 20:843-7. doi: 10.1177/1352458513507822

4. Mori M, Kuwabara S, Paul F. Worldwide prevalence of neuromyelitis optica spectrum disorders. J Neurol Neurosurg Psychiatry. (2018) 89:55556. doi: 10.1136/jnnp-2017-317566

5. Schmidt F, Zimmermann H, Mikolajczak J, Oertel FC, Pache F, Weinhold $\mathrm{M}$, et al. Severe structural and functional visual system damage leads
We conclude that MOG-IgG+ patients in our cohort have more structural brain parenchymal damage, as detected by DTI measures when compared to $\mathrm{HC}$ whereas AQP4-IgG+ patients showed reduced whole brain volumes and reduced volumes of several deep gray matter structures compared to $\mathrm{HC}$. Volume sub-analyses of deep gray matter structures and DTI measurements did not help to differentiate between MOG-IgG+and AQP4-IgG+ patients in our study.

Our results are in favor of an early immunosuppressive treatment of patients with MOG encephalomyelitis whose treatment approaches still are a matter of debate (62). Similar to the treatment strategies for AQP4-IgG+ NMOSD patients, we believe this suggestion is valid because of the increased brain parenchymal damage observed from diffusion tensor imaging measures of parallel diffusivity in MOG-IgG+ patients. Longitudinal imaging studies are warranted to further investigate this trend of increased brain parenchymal damage in MOG$\mathrm{IgG}+$ patients.

\section{DATA AVAILABILITY STATEMENT}

The datasets generated for this study are available on request to the corresponding author.

\section{ETHICS STATEMENT}

The studies involving human participants were reviewed and approved by Charité-Universitätsmedizin Berlin ethics committee (EA1/041/14). The patients/participants provided their written informed consent to participate in this study.

\section{AUTHOR CONTRIBUTIONS}

Study concept and coordination was performed by FP, AB, and MS. Data acquisition and interpretation were performed by FS and MS. Initial draft was performed by FS. Study supervision by $\mathrm{FP}, \mathrm{AB}$, and MS. Critical revision of the manuscript for important intellectual content was performed by FP, MS, AB, CC, KR, JK, NS, JB-S, SA, and SJ. All authors contributed to the article and approved the submitted version. to profound loss of vision-related quality of life in patients with neuromyelitis optica spectrum disorders. Mult Scler Relat Disord. (2017) 11:45-50. doi: 10.1016/j.msard.2016.11.008

6. Finke C, Zimmermann H, Pache F, Oertel FC, Chavarro VS, Kramarenko $\mathrm{Y}$, et al. Association of visual impairment in neuromyelitis optica spectrum disorder with visual network reorganization. JAMA Neurol. (2018) 75:296303. doi: 10.1001/jamaneurol.2017.3890

7. Sepúlveda M, Armangué T, Sola-Valls N, Arrambide G, MecaLallana J, Orecha-Guevara C, et al. Neuromyelitis optica spectrum disorders: comparison according to the phenotype and serostatus. Neurol Neuroimmunol Neuroinflamm. (2016) 3:e225. doi: 10.1212/ NXI.0000000000000225

8. Metz I, Beißbarth T, Ellenberger D, Pache F, Stork L, Ringelstein $M$, et al. Serum peptide reactivities may distinguish neuromyelitis optica subgroups and multiple sclerosis. Neurol Neuroimmunol Neuroinflamm. (2016) 3:e204. doi: 10.1212/ NXI.0000000000000204 
9. Zekeridou A, Lennon VA. Aquaporin-4 autoimmunity. Neurol Neuroimmunol Neuroinflamm. (2015) 2:e110. doi: 10.1212/NXI. 0000000000000110

10. Jarius S, Ruprecht K, Wildemann B, Kuempfel T, Ringelstein M, Geis Ch, et al. Contrasting disease patterns in seropositive and seronegative neuromyelitis optica: a multicenter study of 175 patients. J Neuroinflammation. (2012) 9:14. doi: 10.1186/1742-2094-9-14

11. Sato DK, Callegaro D, Lana-Peixoto MA, Waters P, Jorge F, Takahashi $\mathrm{T}$, et al. Distinction between MOG antibody-positive and AQP4 antibody-positive NMO spectrum disorders. Neurology. (2014) 82:474-81. doi: 10.1212/WNL.0000000000000101

12. Jarius S, Metz I, König FB, Ruprecht K, Reindl M, Paul F, et al. Screening for MOG- IgG and 27 other anti-glial and anti-neuronal autoantibodies in 'pattern II multiple sclerosis' and brain biopsy findings in a MOGIgG-positive case. Mult Scler. (2016) 22:1541-49. doi: 10.1177/13524585 15622986

13. Zamvill SS, Slavin AJ. Does MOG-Ig positive AQP4-seronegative opticospinal inflammatory disease justify a diagnosis of NMO spectrum disorder? Neurol Neuroimmunol Neuroinflamm. (2015) 2:e62. doi: 10.1212/NXI.0000000000000062

14. Narayan R, Simpson A, Fritsche K, Salama S, Pardo S, Mealy M, et al. MOG antibody disease: a review of MOG antibody seropositive neuromyelitis optica spectrum disorder. Mult Scler Relat Disord. (2018) 25:66-72. doi: 10.1016/j.msard.2018.07.025

15. Reindl M, Schanda K, Woodhall M, Tea I, Ramanathan S, Sagen $S$, et al. International multicenter examination of MOG antibody assays. Neurol Neuroimmunol Neuroinflamm. (2020) 7:e674. doi: 10.1212/NXI.0000000000000674

16. Cobo-Calvo A, Indy H, Ruiz A, Collongues N, Kremer L, Durant-Dubief F, et al. Frequency of myelin oligodendrocyte glycoprotein antibody in multiple sclerosis: a multicenter cross-sectional study. Neurol Neuroimmunol Neuroinflamm. (2020) 7:e649. doi: 10.1212/NXI.0000000000000649

17. Jarius S, Ruprecht K, Kleiter I, Borisow N, Asgari N, Pitarokoilio K, et al. MOG-IgG in NMO and related disorders: a multicenter study. Part 1: Frequency, syndrome specificity, influence of disease activity, long-term course, association with AQP4-IgG, and origin. J Neuroinflamm. (2016) 13:279. doi: 10.1186/s12974-016-0717-1

18. Jarius S, Ruprecht K, Kleiter I, Borisow N, Asgari N, Pitarokoilio K, et al. MOG-IgG in NMO and related disorders: a multicenter study. Part 2: Epidemiology, clinical presentation, radiological and laboratory features, treatment, and long-term outcome. J Neuroinflamm. (2016) 13:281. doi: 10.1186/s12974-016-0718-0

19. Jarius S, Ruprecht K, Kleiter I, Borisow N, Asgari N, Pitarokoilio K, et al. MOG-IgG in NMO and related disorders: a multicenter study. Part 3: Brainstem involvement - frequency, presentation and outcome. J Neuroinflamm. (2016) 13:281. doi: 10.1186/s12974-016-0719-Z

20. Pache F, Zimmermann H, Mikolaczak J, Schumacher S, Lacheta A, Oertel FC, et al. MOG-IgG in NMO and related disorders: a multicenter study. Part 4: Afferent visual system damage after optic neuritis in MOG-IgGseropositive versus AQP4-IgG- seropositive patients. J Neuroinflamm. (2016) 13:281. doi: 10.1186/s12974-016-0720-6

21. Cobo-Calvo A, Ruiz A, Maillart E, Audoin B, Zephir H, Bourre $B$, et al. Clinical spectrum and prognostic value of CNS MOG autoimmunity in adults: the MOGADOR study. Neurology. (2018) 90:e1858. doi: 10.1212/WNL.0000000000005560

22. Jurynczyk M, Probert F, Yeo T, Tackley G, Claridge T, Cavey A, et al. Metabolimics reveals distinct, antibody- independent, molecular signatures of MS, AQP4-antibody and MOG-antibody disease. Acta Neuropathol Commun. (2017) 5:95. doi: 10.1186/s40478-017-0495-8

23. Cobo-Calvo A, Sepulveda M, Rollot F, Armangue T, Ruiz A, Maillat E, et al. Evaluation of treatment response in adults with relapsing MOG-Ab-associated disease. J Neuroinflamm. (2019) 16:134. doi: 10.1186/s12974-019-1525-1

24. Ogawa R, Nakashima I, Takahashi T, Kaneko K, Akaishi T, Takai Y, et al. MOG- antibody positive, benign, unilateral, cerebral cortical encephalities with epilepsy. Neurol Neuroimmunol Neuroinflamm. (2017) 4:e322. doi: 10.1212/NXI.0000000000000322

25. Hamid SHM, Whittam D, Saviour M, Alorainy A, Mutzl K, Linaker S, et al. Seizures and encephalitis in myelin oligodentrocyte glycoprotein IgG disease vs AQP4 IgG disease. JAMA Neurol. (2018) 75:65-71. doi: 10.1001/jamaneurol.2017.3196

26. Jarius S, Paul F, Aktas O, Asgari N, Dale RC, DSeze J, et al. MOG encephalomyelitis: international recommendations on diagnosis and antibody testing. J Neuroinflamm. (2018) 15:134. doi: 10.1186/s12974-018-1144-2

27. Cobo-Calvo A, Ayrignac X, Kerschen P, Hourrellou P, Cotton F, Labauge $P$, et al. Cranial nerve involvment in patients with MOG antibody-associated disease. Neurol Neuroimmunol Neuroinflamm. (2019) 6:e543. doi: 10.1212/NXI.0000000000000543

28. Alcaide-Leon P, Cybulsky K, Sankar S, Casserly C, Leung G, Hohol M, et al. Quantitative spinal chord MRI in radiologically isolated syndrome. Neurol Neuroimmunol Neuroinflamm. (2018) 5:e346. doi: 10.1212/NXI.0000000000000436

29. Azevedo CJ, Overton E, Khadka S, Buckley J, Liu S, Sampat $\mathrm{M}$, et al. Early CNS neurodegeneration in radiologically isolated syndrome. Neurol Neuroimmunol Neuroinflamm. (2015) 2:e102. doi: 10.1212/NXI.0000000000000102

30. Pawlitzki M, Neumann J, Kaufmann J, Heidel J, Stadler E, SweeneyReed C, et al. Loss of corticospinal tract integrity in early MS disease stages. Neurol Neuroimmunol Neuroinflamm. (2017) 4:e399. doi: 10.1212/NXI.0000000000000399

31. Van Pelt E, Wong YY, Keteslegers I, Haman D, Hintzen RG. Neuromyelitis optica spectrum disorders: comparison of clinical and magnetic resonance imaging characteristics of AQP4-IgG versus MOG-IgG seropositive cases in the Netherlands. Eur J Neurol. (2016) 23:580-7. doi: 10.1111/ene.12898

32. Kremer S, Renard F, Achard S, Peixoto L, Palace J, Asgari N, et al. Use of advanced magnetic resonance imaging techniques in neuromyelitis optica spectrum disorder. JAMA Neurol. (2015) 72:815-22. doi: 10.1001/jamaneurol.2015.0248

33. Streitberger KJ, Fehlner A, Pache F, Lacheta A, Papazoglou S, Bellmann-Strobl $\mathrm{J}$, et al. Multifrequency magnetic resonance elastography of the brain reveals tissue degeneration in neuromyelitis optica spectrum disorder. Eur Radiol. (2017) 27:2206-15. doi: 10.1007/s00330-016-4561-6

34. Pache F, Zimmermann H, Finke C, Lacheta A, Papazoglu S, Kuchling $\mathrm{J}$, et al. Brain parenchymal damage in neuromyelitis optica spectrum disorder - A multimodal MRI study. Eur Radiol. (2016) 26:441322. doi: 10.1007/s00330-016-4282-X

35. Pasquier B, Borisow N, Rasche L, Bellman-Strobl, Ruprecht K, Niendorf $\mathrm{T}$, et al. Quantitative 7T MRI does not detect occult brain damage in neuromyelitis optica. Neurol Neuroimmunol Neuroinflamm. (2019) 6:e541. doi: 10.1212/NXI.0000000000000541

36. Wingerchuk DM, Banwell B, Bennett JL, Caire P, Carroll W, Chitnis $\mathrm{T}$, et al. International consensus diagnostic criteria for neuromyelitis optica spectrum disorders. Neurology. (2015) 85:177-89. doi: 10.1212/WNL.0000000000001729

37. Schmidt P, Gaser C, Arsic M, Buck D, Förschler A, Berthele A, et al. An automated tool for detection of FLAIR- hyperintense white matter lesions in multiple sclerosis. Neuroimage. (2012) 59:3774-83. doi: 10.1016/j.neuroimage.2011.11.032

38. Yushkevich PA, Piven J, Hazlett HC, Smith G, Ho S, Gee J, et al. User guided $3 \mathrm{D}$ active contour segmentation of anatomical structures: significantly improved efficiency and reliability. Neuroimmage. (2006) 31:1116-28. doi: 10.1016/j.neuroimage.2006.01.015

39. Smith SM, Zhang Y, Jenkinson $M$, Chen J, Matthews PM, Federico A, et al. Accurate, robust, and automated longitudinal and cross-sectional brain change analysis. Neuroimmage. (2002) 17:479-89. doi: 10.1006/nimg.2002.1040

40. Patenaude B, Smith SM, Kennedy DN, Jenkinson M. A Bayesian model of shape and appearance for subcortical brain segmentation. Neuroimmage. (2011) 56:907-22. doi: 10.1016/j.neuroimage.2011.02.046

41. Behrens TE, Woolrich MW, Jenkinson M, Johansen H, Nunei R, Clare S, et al. Characterization and propagation of uncertainty in diffusion-weighted MR imaging. Magn Reson Med. (2003) 50:1077-88. doi: 10.1002/mrm.10609

42. Kuchling J, Backner Y, Oertel F, Raz N, Ruprecht K, Bellman-Strobl J, et al. Comparison of probabilistic tractography and tract based spatial statistics for assessing optic radiation damage in patients with autoimmune inflammatory disorders of the central nervous system. Neuroimage Clin. (2018) 19:53850. doi: 10.1016/j.nicl.2018.05.004 
43. Huak K, Zhang J, Wakana S, Jiang H, Li X, Reich D, et al. Tract probability maps in stereotaxic spaces: analysis of white matter anatomy and tract-specific quantification. Neuroimmage. (2008) 39:336-47. doi: 10.1016/j.neuroimage.2007.07.053

44. R Development Core Team. R: A language and Environment for Statistical Computing. Vienna (2015).

45. Hyun JW, Huh SY, Shin HJ, Woodhall M, Kim S, Irani S, et al. Evaluation of brain distribution criteria at disease onset in differentiating MS from NMOSD and MOG- IgG-associated encephalomyelitis. Mult Scler. (2018) 1:1352458518761186. doi: 10.1177/13524585187 61186

46. Dos Passos GR, Oliveira LM, da Costa BK, Apostolus S, Callegaro D, Fujihara $\mathrm{K}$, et al. MOG-IgG-associated optic neuritis, encephalitis and myelitis: lessons learned from neuromyelitis optica spectrum disorder. Front Neurol. (2018) 9:217. doi: 10.3389/fneur.2018.00217

47. Jurynzcyk M, Geraldes R, Probert F, Woodlhall M, Waters P, Tackley $\mathrm{G}$, et al. Distinct brain imaging characteristics of autoantibodymediated CNS conditions and multiple sclerosis. Brain. (2017) 140:617-27. doi: 10.1093/brain/aww350

48. Jurynzcyk M, Tackley G, Kong Y, Geraldes R, Matthews L, Woodhall $\mathrm{M}$, et al. Brain lesion distribution criteria distinguish MS from AQP4antibody NMOSD and MOG-antibody disease. JNNP. (2017) 88:1326. doi: 10.1136/jnnp-2016-314005

49. Fan M, Fu Y, Su L, Suen Y, Wood K, Yang l, et al. Comparison of brain and spinal cord magnetic resonance imaging features in neuromyelitis optica spectrum disorders patients with or without aquaporin-4 antibody. Mult Scler Relat Disord. (2017) 13:58-66. doi: 10.1016/j.msard.2017. 02.003

50. Waters P, Reindl M, Saiz A, Schandu K, Tuller V, Kral V, et al. Multicenter comparison of a diagnostic assay: aquaporin-4 antibodies in neuromyelitis optica. J Neurol Neurosurg Psyychiatry. (2016) 87:100515. doi: 10.1136/jnnp-2015-312601

51. Melamed E, Levy M, Waters PJ, Sato D, Bennett J, John G. Update on biomarkers in neuromyelitis optica. Neurol Neuroimmunol Neuroinflamm. (2015) 2:e134. doi 10.1212/NXI.00000000000 00134

52. Kitley J, Waters P, Woodhall M, Leite I, Murchison A, George J, et al. Neuromyelitis optica spectrum disorders with aquaporin-4 and myelinoligodentrocyte glycoprotein antibodies. A comparative study. JAMA Neurol. (2014) 71:276-83. doi: 10.1001/jamaneurol.2013.5857

53. Sun SW, Liang HF, Schmidt R, Cross A, Song S. Selective vulnerability of cerebral white matter in a murine model of multiple sclerosis detected using diffusion tensor imaging. Neurobiol Dis. (2007) 28:308. doi: 10.1016/j.nbd.2007.06.011

54. Budde MD, Xie M, Cross AH, Song S. Axial diffusivity is the primary correlate of axonal injury in the experimental autoimmune encephalomyelitis spinal chord: a quantitative pixelwise analysis. J Neurosci. (2009) 29:280513. doi: 10.1523/JNEUROSCI.4605-08.2009

55. Kolbe S, Bajrazewski C, Chapman C, Nguyen T, Mitchell P, Paine M, et al. Diffusion tensor imaging of the optic radiations after optic neuritis. Hum Brain Mapp. (2012) 33:2047-61. doi: 10.1002/hbm.21343

56. Naismith RT, Xu J, Tutlam NT, Lancia S, Trinkaus K, Song S, et al. Diffusion tensor imaging in acute optic neuropathies: predictor of clinical outcomes. Arch Neurol. (2012) 69:65-71. doi: 10.1001/archneurol.2011.243

57. Aung WY, Mar S, Benzinger TL. Diffusion tensor MRI as a biomarker in axonal and myelin damage. Imaging Med. (2013) 5:427-40. doi: 10.2217/iim.13.49

58. Kolbe S, Chapman C, Nguyen T, Bajraszewski C, Johnston L, Kean $M$, et al. Optic nerve diffusion changes and atrophy jointly predict visual dysfunction after optic neuritis. Neuroimage. (2009) 45:679-86. doi: 10.1016/j.neuroimage.2008.12.047

59. Finke C, Heine J, Pache F, Lacheta A, Borisow N, Kuchling J, et al. Normal volumes and microstructural integrity of deep gray matter structures in AQP4+ NMOSD. Neurol Neuroimmunol Neuroinflamm. (2016) 3:e229. doi: 10.1212/NXI.0000000000000229

60. Vanikieti K, Poonyathalang P, Jindhara P, Ladeta A, Fujihara K, Prayoonvivat $\mathrm{N}$, et al. Clinical characteristics and long-term visual outcome of optic neuritis in neuromyelitis optica spectrum disorder: a comparison between Thai and American- Caucasian cohort. Mult Scler Relat Disord. (2017) 17:8791. doi: 10.1016/j.msard.2017.07.013

61. Hor J, Asgari N, Nakashima I, Broadley S, Leite I, Kissani N, et al. Epidemiology of neuromyelitis optica spectrum disorder and its prevalence and incidence worldwide. Front Neurol. (2020) 11:501. doi: 10.3389/fneur.2020.00501

62. Borisow N, Mori M, Kuwabara S, Scheel M, Paul F. Diagnosis and treatment of NMO spectrum disorder and MOG-Encephalomyelitis. Front Neurol. (2018) 9:888. doi: 10.3389/fneur.2018.00888

Conflict of Interest: FS served on the scientific advisory board of Novartis, received travel funding and/or speaker honoraria from Biogen Idec, Bayer Healthcare, Teva Pharmaceuticals and Sanofi-Aventis/Genzyme. JK received conference registration fees from Biogen and financial research support from Krankheitsbezogenes Kompetenznetzwerk Multiple Sklerose (KKNMS), not related to this work. JB-S received travel funding and speaking fees from Bayer Healthcare, Sanofi-Aventis/Genzyme, and Teva Pharmaceuticals. KR served on the scientific advisory board for Sanofi-Aventis/Genzyme, Novartis, and Roche; received travel funding and/or speaker honoraria from Bayer Healthcare, Biogen Idec, Merck Serono, Sanofi-Aventis/Genzyme, Teva Pharmaceuticals, Novartis, and Guthy Jackson Charitable Foundation; is an academic editor for PLoS ONE; receives publishing royalties from Elsevier; and received research support from Novartis and German Ministry of Education and Research. SA received travel funding from Celgene. $\mathrm{AB}$ served on the scientific advisory board for Biogen; received travel funding and/or speaker honoraria from Novartis and Biogen; has patents pending from method and system for optic nerve head shape quantification, perceptive visual computing based postural control analysis, multiple sclerosis biomarker, and perceptive sleep motion analysis; has consulted for Nexus and Motognosis; and received research support from Novartis Pharma, Biogen Idec, BMWi, BMBF, and Guthy Jackson Charitable Foundation. FP serves on the scientific advisory board for Novartis; received speaker honoraria and travel funding from Bayer, Novartis, Biogen Idec, Teva, Sanofi-Aventis/Genzyme, Merck Serono, Alexion, Chugai, MedImmune, and Shire; is an academic editor for PLoS ONE; is an associate editor for Neurology $($ Neuroimmunology \& Neuroinflammation; consulted for SanofiGenzyme, Biogen Idec, MedImmune, Shire, and Alexion; and received research support from Bayer, Novartis, Biogen Idec, Teva, Sanofi-Aventis/Genzyme, Alexion, Merck Serono, German Research Council, Werth Stiftung of the City of Cologne, German Ministry of Education and Research, Arthur Arnstein Stiftung Berlin, EU FP7 Framework Program, Arthur Arnstein Foundation Berlin, Guthy Jackson Charitable Foundation, and National Multiple Sclerosis of the USA. MS holds a patent for manufacturing of phantoms for computed tomography imaging with 3D printing technology and received research support from Federal Ministry of Economics and Technology.

The remaining authors declare that the research was conducted in the absence of any commercial or financial relationships that could be construed as a potential conflict of interest.

The reviewer ML-P declared a past co-authorship with one of the authors FP to the handling editor.

Copyright (C) 2020 Schmidt, Chien, Kuchling, Bellmann-Strobl, Ruprecht, Siebert, Asseyer, Jarius, Brandt, Scheel and Paul. This is an open-access article distributed under the terms of the Creative Commons Attribution License (CC BY). The use, distribution or reproduction in other forums is permitted, provided the original author(s) and the copyright owner(s) are credited and that the original publication in this journal is cited, in accordance with accepted academic practice. No use, distribution or reproduction is permitted which does not comply with these terms. 